\title{
Impact of digital information systems on supply chain performance: a mediation of integrations and green practices
}

\author{
Syed Abdul Rehman Khan ${ }^{1,2,3, *}$, Zhang $\mathrm{Yu}^{4}$, Adeel $\mathrm{Shah}^{5}$, and Muhammad Tanveer ${ }^{6}$ \\ ${ }^{1}$ School of Management and Engineering, Xuzhou University of Technology, Xuzhou, China \\ ${ }^{2}$ Department of Business Administration, ILMA University, Karachi, Pakistan \\ ${ }^{3}$ Beijing Key Laboratory of Urban Spatial Information Engineering, Beijing, China \\ ${ }^{4}$ School of Economics and Management, Chang'an University, Xi'an, China \\ ${ }^{5}$ Collage of Business Management (CBM), Karachi, Pakistan \\ ${ }^{6}$ Prince Sultan University, Rafha Street, Riyadh 11586, Saudi Arabia
}

\begin{abstract}
Industry Revolution (IR) 4.0 is revolutionizing the information infrastructure and the systems in supply chain management. However, the employment of the new technology is not sufficient to impact Supply Chain Management Performance (SCMP). Organizations need to build capabilities like integration both Upstream (UI) and Downstream (DI) for the technology to increase SCMP. The Green Practices (GP) are also becoming part of global corporate culture as all stakeholders demand sustainability in the value chain. The study tests IR 4.0, UI, DI, and GP to understand its impact and relationship with SCMP. For the statistical testing conducts on primary data collected from Malaysian firms. AMOS structural equation model is used to test the model and infer the results. The test concludes that IR 4.0 along with UI, DI, and GP impacts SCMP positively.
\end{abstract}

Keywords: Industry revolution 4.0, Green practices, Supply chain management performance, Malaysian firms.

\section{Introduction}

IR 4.0 is the revolution of technology information systems enveloping advanced applications to manage business operations. The technology enables businesses to create, collect and process data into meaningful information for decision making [1] . The data is collected from within a business organization and external sources. Data sources can be many for a manufacturing unit, including machines, organizational functions, and human and machine interfaces [2]. Such massive data requires advanced infrastructure for realtime data sharing and a central data processing unit to handle high data influx. The IR 4.0 requires multiple sensors, gauges, network apparatus, and other fast devices to create, share and collect data at high speed. The information updates are happening in real-time, thus

${ }^{*}$ Corresponding author: sarehman $\operatorname{cscp} @$ yahoo.com 
enabling the organization to improvise and become agile according to market needs. The IR 4.0 is playing a pivotal role in assisting in increasing supply chain performance.

The technology adoption by organizations is not sufficient to increase supply chain performance. Both tangible and nontangible capability combines to influence performance. One of the essential nontangible capabilities which are most important in the supply chain is integration between organizations. Organizational integration enables money, material, and information to flow without any hindrance in the supply network. The supply network has two ends: at one end, supplier exists, which refers to Upstream, and the customer's location in the supply chain is known as downstream. The integration entails both upstream and downstream integration. The upstream integration refers to assimilation between supplier and manufacturer. Whereas downstream envelopes manufacturer and customer integration. The concept revolves around material, money, and information sharing between organizations on both supply chain ends. All type of integration needs the amicable culture and top management support for an alignment and synergy. The idea of integration differs from collaboration which entails both horizontal and vertical cooperation between organizations. In integration, the organizational boundaries remain black and white while systematically infusing to transmit signals (information) and flow of money and material in the supply chain: this requires culture, training, policies, platform, and clear understanding of customer needs which supply network exists to satisfy. Technology is only providing the venue, whereas other factors mentioned are equally crucial for integration to occur. Without integration in both directions, the supply chain would cease to exist, or the lack might create inefficiencies or disruption of material, money, and information.

Further to increasing supply chain performance, green practices also play an essential role. The green practices in the supply chain target to improve the sustainability of the whole supply chain. The absence of sustainability could dent the environment and negatively impact the triple bottom line (planet, people, and profit). The green practices envelop all logistics drivers: inventory, transportation, and facility (warehouse, factories, and cross-docking). Besides the conventional performance of the supply chain relating to operations, sustainability is also an essential performance dimension in the modern world. The sustainability compulsions in the contemporary era are due to United Nations Sustainability 17 goals for 2030 [3]. The United Nations sustainability goals compel all governments around the globe to enact laws and regulations to increase socioeconomic gains. Besides being governmental regulations, sustainability is much demanded by customers as it results to improve business performance. Thus, the customer and other stakeholders' demand for sustainability paved the way to introduce green practices in the supply chain.

Based on the importance of digital information systems, upstream and downstream integration, and green practices: the factors are combined in the research to evaluate their relationship in the context of supply chain performance. The unique combination of the variables that have significant importance in academic writing would realize insight beneficial for practitioners and researchers. The following sections in the research bring forward the literature review of the selected variables to propose hypothesis testing. The methodology includes data collection, statistical tests used for analysis, and discussion on the results. Lastly, the research concludes with policy implications based on arrived results.

\section{Literature review}

\subsection{IR4.0 and SCMP}

The advanced supply chain networking system like IR 4.0 has revolutionized the 
responsiveness of inter-organizations. The organizational responsiveness to the market has multiplied as information amongst the supply chain partners is shared in real-time [4]. According to [5], advanced supply chain systems on information contribute to IR 4.0, influencing performance. [5], in their research-tested IR 4.0 on performance based on 205 responses from different businesses in India. The data-driven systems help to achieve an organizational competitive advantage, thus contributing to the whole value chain. A similar claim for information sharing technology like IR 4.0 to influence SCMP was made by [6]. The researchers collected primary data of 285 responses to test their hypothesis. Based on evidences found in literature that the two variables have been tested earlier in different contexts support to propose below hypothesis:

H1: IR 4.0 is positively correlated with SCMP

\subsection{Directional integration mediates IR4.0 and SCMP}

For network systems to work effectively, close association with downstream and upstream is essential for information sharing [1]. According to [1], the sharing of information between supply chain partners at both ends is based on trust and relationship. [1] empirically tested primary data of 261 responses to test if the integration along supply chain enablers mediates between technology like IR 4.0 and performance: Confirmatory Factor Analysis and Structural Equation Modelling (SEM) performed. The relationship must be robust to affect information sharing between the partners. There are reported incidents where inter-organizational employees have shown reluctance due to a lack of training and the proper procedure. However, many researchers claimed that training plays an integral part in the successful implementation of Industry 4.0 [7]. Once the right culture, procedures, and training, downstream and upstream integration would make money, material, and information flow without any hindrance. According to [8], integration remains an essential factor in the context of IR 4.0. The study collected data from 380 retail respondents to test their proposed model. The SEM testing confirms the study's proposed hypothesis, which claims that integration increases SCMP and is significant IR 4.0.

Similarly, [9] also brought forward the importance of integration to implement IR 4.0 and be pivotal for the technology to succeed. [9] conducted an in-depth analysis using the case study methodology on a manufacturing firm. Their finding also remains conclusive that for successful IR 4.0 implementation, integration remains of utmost importance. Thus, it concludes that for implementing IR 4.0 to impact supply chain performance, downstream and upstream integrations are the critical enabler that mediates to affect supply chain performance. Based on the above literature evidence, below are the derived hypothesis:

H2: UI mediates the relationship between IR 4.0 and SCMP

H3: DI mediates the relationship between IR 4.0 and SCMP

\subsection{GP mediates the relationship between IR 4.0 and SCMP}

Green practices in supply chain management have played a crucial role in impacting sustainability and the firm's economic performance. The created impact by green practices transduces from firm to supply chain network, thus giving a more profound effect on customer and stakeholder satisfaction. The same point has been raised by [10] where they tested GP mediation for between technology and Firm performance. The study verified a full mediation effect of GP between technology related to information and firm socialeconomic performance. The research bases on primary data: 284 responses (manufacturing firms). Smart-PLS was used for testing the proposed research hypothesis. According to [8], supply chain performance strongly relates to a firm's performance. The study in its future direction suggests that GP should be tested to check its impact on SMCP. The GP entails all 
logistical drivers (transportation, facility, and inventory), about material and money. Transportation impacts directly to the environment due to engine exhaustion because of burning of fossil fuel. In order to employ green practices, companies are reducing carbon footprint either by using electric car or developing efficient engines for lesser carbon footprint. Besides transportation inventory is also has a important role to play in sustainability as its waste can impact environment: as virgin raw material extraction uses a lot of energy and finances. In between IR4.0 and SCMP, GP has an important role which ensures sustainability and economic gains to organization and supply chain at large. Thus it could concludes that GP indirectly helps technology to influence supply chain performance. Based on the above theoretical evidence, we proposed the following hypothesis:

H4: GP mediates the relationship between IR 4.0 and SCMP

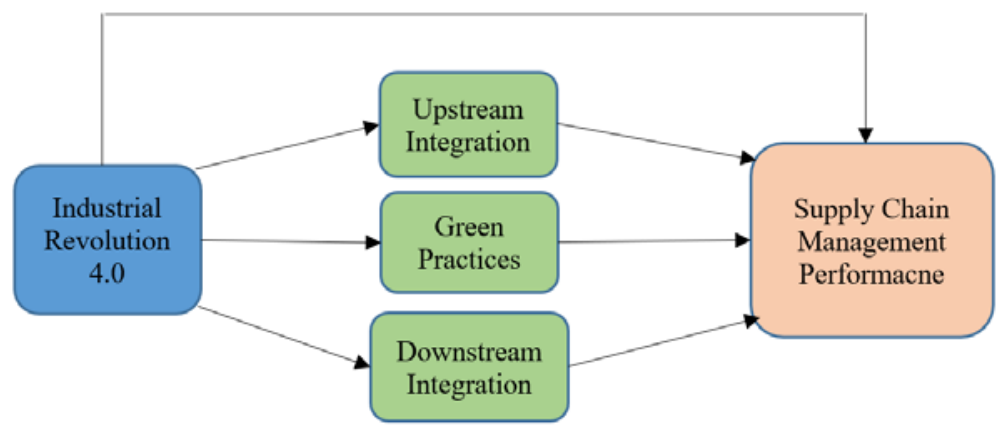

Fig. 1. Research model.

\section{Methodology and data collection}

The research aims are to analyze IR 4.0 impact on SCMP and mediation of DI, UI, and GP in the Malaysian industry. A total of 430 organizations were selected for the distribution of questionnaires to collect responses. The questionnaire covered all the variables selected for the study. The questionnaire was distributed using a google form to which respondents selected scores 1 to 5 on the Likert scale. The response rate was 69. 06\%: the received questionnaire was 326, out of which 29 were removed due to inconsistency. The testing of the hypothesis was conducted on 297 responses. AMOS SEM was used to test the hypothesis.

\section{Results and discussion}

The hypothesis results are empirically tested through AMOS SEM, which are displayed in table 2. The H1 was not supported; hence, it is concluded that IR 4.0 does not correlate with SCMP, similar to [6]. Their study tested the relation between digital technology for information sharing; IR 4.0 does not relate to SCMP directly.

However, H2, H3, and H4 hypotheses tested for mediation were conclusive to support. $\mathrm{H} 2$ and H3 proposed to mediate UI and DI between IR 4.0 and SCMP. The results are consistent with [8], in which integration and alliances were found to impact the relationship between IR 4.0 and SCMP. Lastly, the H4 value is consistent with [5], which concluded their results confirming the GP fully mediates IR 4.0 and SCMP.

Table 1. Hypothesis results. 


\begin{tabular}{|c|c|c|c|c|}
\hline Hypothesis & Relationship & Path coefficient & P-value & Decision \\
\hline \multicolumn{5}{|c|}{ Direct Effect } \\
\hline H1 & IR 4.0 $>$ SCMP & 0.751 & 0.251 & Not support \\
\hline \multicolumn{5}{|c|}{ Mediation } \\
\hline H2 & UI x IR4.0 $>$ SCMP & 0.122 & 0.000 & Support \\
\hline H3 & DI x IR4.0 $>$ SCMP & 0.182 & 0.000 & Support \\
\hline H4 & GP x IR4.0 $>$ SCMP & 0.541 & 0.000 & Support \\
\hline
\end{tabular}

\section{Conclusion}

Supply Chain information networks play an essential role in connecting inter-organizations in supply chain management. The modern supply chains are data-driven and require to share of data in real-time. The real-time connectivity is only possible with implementing IR 4.0 in the supply chain; however, as the results conclude, it does not directly impact the SCMP but requires more organizational capabilities to mediate between variables. The study found that such capabilities are DI, UI, and GP. The mediating variables selected are all intangible capabilities that organizations can create via the proper operational procedures, culture, training, top management support, and alignment between interorganizational. The integration in both directions will allow IR 4.0 to work efficiently to fulfill its purpose. The information-sharing could only be possible if stakeholders in the chain are assimilated for a common goal to satisfy the customer. At the same time, GP is a set of rules, regulations, mindset, and understanding about sustainability and its advantages. Once the organization's human resources in the supply chain collectively work together to practice green supply functions, that can surely assist and set the direction of IR 4.0 to impact SCMP. The implementation of IR 4.0, directional integrations, and GP requires direct attention from top management. Their influence will enable the right culture for a permanent and everlasting supply chain performance, thus resulting in true customer satisfaction.

This research is supported by the Beijing Key Laboratory of Urban Spatial Information Engineering (NO. 20210218) and China's National Natural Science Foundation (No. 202202457).

\section{References}

1. Cadden, T., Dennehy, D., Mantymaki, M., \& Treacy, R. (2021). Understanding the influential and mediating role of cultural enablers of AI integration to supply chain. International Journal of Production Research, 1-29. https://doi.org/10.1080/00207543.2021.1946614

2. Rossit, D. A., Tohmé, F., \& Frutos, M. (2019). A data-driven scheduling approach to smart manufacturing. Journal of Industrial Information Integration, 15, 69-79.

3. United Nations. (n.d.). United Nations Sustainable Development - 17 Goals to Transform Our World. 2021. Retrieved May 21, 2021, from https://www.un.org/sustainabledevelopment/

4. Lin, D., Lee, C. K. M., Lau, H., \& Yang, Y. (2018). Strategic response to Industry 4.0: an empirical investigation on the Chinese automotive industry. Industrial Management \& Data Systems.

5. Kamble, S., Gunasekaran, A., \& Dhone, N. C. (2020). Industry 4.0 and lean manufacturing practices for sustainable organisational performance in Indian 
manufacturing companies. International Journal of Production Research, 58(5), 13191337.

6. Tigga, G. A., Kannabiran, G., \& Arumugam, V. (2021). Exploring relationships among IT advancement, IT assimilation, supply chain capabilities and supply chain performance. Journal of Decision Systems, 1-25. https://doi.org/10.1080/12460125.2021.1873022

7. Moeuf, A., Lamouri, S., Pellerin, R., Tamayo-Giraldo, S., Tobon-Valencia, E., \& Eburdy, R. (2020). Identification of critical success factors, risks and opportunities of Industry 4.0 in SMEs. International Journal of Production Research, 58(5), 1384-1400.

8. Gawankar, S. A., Gunasekaran, A., \& Kamble, S. (2020). A study on investments in the big data-driven supply chain, performance measures and organisational performance in Indian retail 4.0 context. International Journal of Production Research, 58(5), 1574-1593. https://doi.org/10.1080/00207543.2019.1668070

9. Ghobakhloo, M., \& Fathi, M. (2020). Corporate survival in Industry 4.0 era: the enabling role of lean-digitized manufacturing. Journal of Manufacturing Technology Management, 31(1), 1-30. https://doi.org/10.1108/JMTM-11-2018-0417

10. Umar, M., Khan, S. A. R., Yusoff Yusliza, M., Ali, S., \& Yu, Z. (2021). Industry 4.0 and green supply chain practices: an empirical study. International Journal of Productivity and Performance Management, ahead-of-p(ahead-of-print). https://doi.org/10.1108/IJPPM-12-2020-0633 